\title{
First study in cryopreserved Crassostrea angulata sperm
}

\author{
Riesco Marta F. ${ }^{1}$, Félix Francisca ${ }^{1}$, Matias Domitilia ${ }^{2}$, Joaquim Sandra ${ }^{2}$, Suquet Marc ${ }^{3}$, \\ Cabrita Elsa ${ }^{1,{ }^{*}}$
}

${ }^{1}$ CCMAR, University of Algarve, Campus of Gambelas, 8005-139 Faro, Portugal

2 IPMA, IP, Av.5 de Outubro s/n, 8700-305 Olhão,Portugal

${ }^{3}$ IFREMER, UMR 6539 LEMAR, Departement PFOM/Laboratoire ARN Station Expérimentale

d'Argenton, Presqu'île du Vivier, 29840 Argenton, France

* Corresponding author :Elsa Cabrita, Tel.: +351.289800900 ext 7532; fax: +351.289800069. ;

email address : ecabrita@ualg.pt

\begin{abstract}
:
Sperm cryopreservation is a widely employed technique that promotes alternative techniques to contribute to broodstock management or restoration programs for species of commercial interest, endangered species or species with an interesting genotype. The preservation of genetic material from improved stocks or from the original population is extremely important for the oyster aquaculture industry to prevent the potential impacts of epidemic diseases and natural disasters. The Portuguese oyster, Crassostrea angulata, was the most important species commercialized by the shellfish industry. However, inadequate management of this industry and pathology occurrences resulted in a significant decrease in natural populations. For this reason, in this work a successful sperm cryopreservation protocol for this important species has been developed for the first time. Different internal cryoprotectants (DMSO, ethylene glycol, polyethylene glycol and methanol) at several concentrations $(5,10,20 \%)$, containers (straws vs cryovials) and freezing rates (slow and fast rates) were tested. Cryoprotectant toxicity tests corroborated that this assay did not take into account the following steps of cryopreservation protocol as sperm agglutination. A fast freezing rate of cells diluted in $10 \%$ DMSO and the use of straws as containers were the best cryopreservation conditions for Portuguese oyster sperm. Finally, fertilization assays confirmed the efficiency of the cryopreservation protocol in oyster sperm. These results demonstrated that different susceptibilities have been detected concerning sperm cryopreservation depending on oyster species or genetic material composition.
\end{abstract}

\section{Highlights}

- Toxicity tests do not take into account the following steps of cryopreservation protocol as sperm agglutination. 10\% DMSO, fast freezing and the use of straws as container represent the best cryopreservation conditions for Portuguese oyster sperm. - Fertilization ability is the definitive tool to evaluate the cryopreservation protocol efficiency in oyster sperm. - Sperm cryopreservation protocol was successfully established in $C$. angulata for the first time.

Keywords : Crassostrea angulata, Sperm, Cryopreservation, Motility, Viability, Fertilization 


\section{Introduction}

Most bivalve production still relies on the collection of wild seed (Suquet et al., 2014). Seed collection can have a negative environmental impact or be a limiting factor in the development of the industry due to the unpredictable seed supply (Corporeau et al., 2012). Seed production in hatcheries constitutes a complement to the wild seed supply for the shellfish industry, and can offer new opportunities for genetic improvement, including the creation of special lines (e.g. tetraploids, disease resistant). Moreover, a number of bivalve species worldwide, including oysters, have been affected by mass mortality events associated with herpesviruses (European Food Safety Authority, EFSA, 2015), resulting in significant losses. In this respect, the development of selection programs to obtain improved selected genotypes combined with mid- and long-term cryopreservation in cryobanks of these genotypes could be a successful tool for restoration programs in some endangered species or populations.

In this regard, sperm cryopreservation as a tool for preserving genetic material has already been established in several fish and oyster species. These cryobanks allow the availability of gametes all year round for fertilization outside the normal reproductive seasons (Hu et al., 2011; Yang et al., 2012). In terms of sperm cryopreservation, oysters are the most widely studied mollusc species, in particular the Pacific oyster, Crassostrea gigas (Hassan et al., 2015). There are other species of particular interest in which cryopreservation could play a particular role in the conservation of native populations and in improving reproductive strategies for 
production. In the Portuguese oyster, $C$. angulata, sperm has not been cryopreserved until now. Some decades ago in Europe, C.angulata was the major species commercialized by the shellfish industry (Boudry et al., 1998). However, inadequate management of this industry, together with diminished water quality, exposed the species to several pathologies that almost lead to its extinction (Comps et al., 1976). Nowadays, pure wild populations of $C$. angulata are rare to find. Cryopreservation technology could promote alternative techniques to contribute to the management of restoration programs in this important species. Although protocols have been developed for a closely related species, C. gigas, different susceptibilities have been detected concerning sperm cryopreservation depending on oyster species or genetic material composition (Dong et al., 2011). Therefore, the direct application of one protocol to a wider number of species may cause a decrease in sperm cryopreservation success due to variability in the cryopreservation process (sperm collection, selection of extenders, concentration and type of cryoprotectants (CPAS), packaging of sperm and freezing and thawing rates) within and among species (Tiersch, 2000).

For this reason, the aim of the present work was to establish a sperm cryopreservation protocol for $C$. angulata sperm for the first time. Previous studies in different oyster species were taken into account, and some preliminary assays, such as caffeine addition (Suquet et al., 2012) were carried out for the improvement of postthaw sperm motility.

\section{Material and methods}




\subsection{Oyster maintenance}

Oyster broodstock (C. angulata) with a commercial weight $>70 \mathrm{~g}$ was acquired from the Ria de Alvor and Mira river bivalve farms during the natural spawning season and maintained at the Experimental Shellfish Aquaculture Station (IPMA, I.P.), Portugal. During this period, oysters were fed daily with a mixture of two microalgae (Chaetoceros calcitrans and Skeletonema costatum: 50/50 cells/ $\mu \mathrm{L}$ ) and kept in open systems. Temperature was maintained at $20^{\circ} \mathrm{C}$ to $22^{\circ} \mathrm{C}$.

\subsection{Sperm collection}

The oysters were opened and a small sample of gonad tissue was examined microscopically to determine sex. Sperm was collected by the dry method, extracting the sperm directly from the gonad using a micropipette. The gonadal area was previously wiped to remove any contamination. Depending on the experiment, sperm was pre-diluted in an extender media and maintained at 4 으 $\mathrm{C}$ until used. Sperm from at least 3 males was used in all the experiments.

\subsection{Effect of the extender solution}

In order to test two extender solutions for $C$. angulata oyster sperm, samples were collected as previously described and immediately pre-diluted 1:10 (v/v) in artificial sea water (Asw) (Gwo et al., 2002) or in Asw supplemented with $10 \mathrm{mM}$ caffeine $(\mathrm{pH}=8.1$; osmolarity: $1150 \mathrm{mOsm} / \mathrm{Kg}$ ). The diluted sperm was filtered using two different sieves: $100 \mu \mathrm{m}$ to retain larger impurities such as gonad clumps, and $20 \mu \mathrm{m}$ for smaller debris. The samples were stored at 4 으 for 3 days. Motility was analyzed daily until the end of the experiment. Prior to this, a set of experiments was performed to test the effect of sperm temperature during activation (Figure 1 Supplementary Material). Thus, sperm 
motility was analyzed further by waiting 30 minutes at RT (Room Temperature) before loading the sperm into the Makler chamber and activating it with Asw or Asw+caffeine.

Total motility was determined using ISAS software (ISAS, Proiser R+D, S.L., Spain). Using a $10 \mu \mathrm{L}$ sample, sperm movement and concentration were determined using CASA software. For all the analyses, motility was assessed in a Makler chamber using a phase-contrast microscope (Nikon 200, Japan) with a $10 \times$ negative contrast objective, and a digital camera (Basler A312f C-mount, Germany) set for $25 \mathrm{fps}$. The CASA software settings were adapted to oyster sperm. The parameter settings were: 1 < Particles Area (in microns ${ }^{2}$ ) < 90; connectivity: 6 . Only males showing total motility rate values equal to or higher than $40 \%$ were used. Sperm concentration was measured using the same software and values ranged from 1 to $2 x$ $10^{9}$ spermatozoa/ml in all analyzed samples. The experiments were performed using 4 individual males.

\subsection{Cryoprotectant toxicity assays}

The aim of the toxicity test was to determine the best cryoprotectants (CPAs) to use and their ideal concentration. For this, four different CPAs were tested, polyethylene glycol (PEG), dimethyl sulfoxide (DMSO), ethylene glycol (EG) and methanol (MetOH), at three concentrations $(5,10$ and $20 \%, v / v$ final concentration) and in two dilution mediums, Asw and Asw supplemented with $10 \mathrm{mM}$ caffeine. Sperm motility and viability were the analyzed parameters chosen in the toxicity study.

Pre-diluted sperm was diluted 1:1 (v/v) under each condition and exposed to the CPAs effect for 10 minutes (equilibration time) at $4^{\circ} \mathrm{C}$. After this, $10 \mu \mathrm{L}$ of diluted sperm were used to determine motility as previously described, and $15 \mu \mathrm{L}$ were used for the 
viability test. The percentage of motile cells and cell concentration were recorded. Spermatozoa viability was determined mixing $15 \mu \mathrm{L}$ of diluted sperm, $0.5 \mu \mathrm{L}$ SYBR Green (final concentration $100 \mathrm{nM}$ ) and $1.5 \mu \mathrm{L}$ propidium iodide (final concentration $12 \mu \mathrm{M}$ ) and observed in a fluorescence microscope (Nikon E200, Japan) equipped with a triple excitation filter block DAPI-FITC-Texas Red (excitation filter wavelengths: 395$410 \mathrm{~nm}$ (bandpass, $403 \mathrm{CWL}), 490-505 \mathrm{~nm}$ (bandpass, $498 \mathrm{CWL}$ ), and 560-580 $\mathrm{nm}$ (bandpass, $570 \mathrm{CWL}$ ). The percentage of live cells (cells with membrane integrity-green cells) was recorded. At least 100 cells per slide were counted, and two slides per pool and per treatment were observed. Both motility parameters and cell viability were measured in fresh sperm as the control. The lowest toxicity conditions were chosen for the next steps ( $n=3$ individual males).

\subsection{Cryopreservation assays}

\subsubsection{Comparison of two different freezing rates}

In this experiment, two different freezing rates were tested. Sperm was diluted in DMSO and PEG at two concentrations (10 and 20\%), and diluted in Asw or Asw+ 10 $\mathrm{mM}$ caffeine. The conditions for the slower freezing were: $0.3^{\circ} \mathrm{C} / \mathrm{min}$ from 0 to $-30{ }^{\circ} \mathrm{C}$, after which the samples were plunged directly into liquid nitrogen, and for the faster freezing, $6{ }^{\circ} \mathrm{C} / \mathrm{min}$ from 0 to $-70{ }^{\circ} \mathrm{C}$ also followed by plunging the samples directly into liquid nitrogen. The freezing rates were adapted from those previously employed by other authors in C. gigas (leropoli et al.,2004; Suquet et al., 2014). A portable programed biofreezer (Asymptote Grant EF600, UK) was used for both freezing rates. All samples were cryopreserved in $0.5 \mathrm{~mL}$ French straws (IMV, France). 
For thawing, the straws were removed from the liquid nitrogen and placed in a water bath at $37^{\circ} \mathrm{C}$ for $10 \mathrm{~s}$ and the samples were immediately used to evaluate sperm quality, through motility and viability tests, as described before. Samples presenting agglutination were discarded after thawing in order to preserve sperm viability. Agglutination was verified by analyzing photos of cryopreserved samples (Canon G12 digital camera) and classified according to the method described by Dong et al., (2007). This method was based on an agglutination scale with five different levels of sperm agglutination: (i) homogeneous suspension, level 0; (ii) few clumps discernable, level 1; (iii) many clumps evident, level 2; (iv) aggregation of clumps, level 3; (v) formation of elongated clumping ("noodles"), level 4; (vi) formation of well-developed noodles, level 5. After the photographic evaluation, viability photos were acquired from the same samples in order to determine whether agglutination correlated with cell survival.

All the experiments were repeated using at least three different males and the same samples were analyzed in fresh as the control.

\subsubsection{Establishment of sperm packaging: straws vs cryovials}

The type of container was also tested using $0.5 \mathrm{~mL}$ French straws and $1 \mathrm{~mL}-$

cryovials. To cryopreserve the samples, the best conditions obtained previously were chosen. Samples were diluted in Asw containing 10\% DMSO and the faster freezing rate was used. The comparison between straws and cryovials was based on the motility and viability results. 
Samples cryopreserved in straws and cryovials were thawed at $37^{\circ} \mathrm{C}$ during 10 and $90 \mathrm{~s}$, respectively. These different times were established according to the sample volume, sperm concentration, and container type.

\subsubsection{Fertilization capacity}

Fertilization capacity was tested in order to evaluate the designed protocol. For fertilization procedures both gametes were extracted using two different methods. Male gametes were obtained by the dry method, as described before, and female gametes by the wet method. In the wet method, the oocytes were released by the gonad, using a scalpel, performing small horizontal and vertical cuts. Artificial seawater was used to wash the gonad and to collect the oocytes into a $1 \mathrm{~L}$ beaker. For each fertilization, gametes from at least four different females were collected and pooled in equal amounts. The oocytes were then filtered using two sieves: $100 \mu \mathrm{m}$ to retain larges debris and $20 \mu \mathrm{m}$ to retain the oocytes, and left to incubate in $1 \mathrm{~L}$ Asw for $30 \mathrm{~min}$ to induce final maturation. Oocyte concentration was determined counting 5 different $1 \mathrm{~mL}$ subsamples from each pool $(n=5)$ in a counting chamber (Sedgewick rafter, Portugal). Calculations for the fertilization procedures were made in order to have 200 000 eggs/L for each incubator.

For the sperm, approximately 40 straws per pool (number of males per pool=3 and number of pools $=5)$ were thawed using the conditions previously established $(10 \%$ DMSO in Asw, and fast freezing method) and an aliquot was removed from the cryopreserved samples to determine concentration after thawing, using the CASA system. The same procedure was followed for the fresh samples. Four different spermatozoa/egg ratios (1 000, 2 500, 5000 and $10000 \mathrm{spz} / \mathrm{egg}$ ) were tested for the 
cryopreserved material. For the control (pre-diluted fresh sperm in Asw), a ratio of 1000 spz/egg was used according to Song et al. (2009) for C.gigas. For each fertilization, oocytes were maintained in $500 \mathrm{~mL} \mathrm{Asw}$ and the correct proportion of sperm was added and mixed. After 20-30 min, $500 \mathrm{~mL}$ of fresh seawater was added to the $1 \mathrm{~L}$ beaker. Fertilizations were done using 5 replicates under controlled environmental conditions and kept at $21-22{ }^{\circ} \mathrm{C}$ for 24 hours. Five subsamples of eggs were removed after homogenization and the number of fertilized and non-fertilized eggs counted. The percentage of fertilized eggs was calculated in respect to the total number of incubated eggs.

\subsection{Statistical analysis}

Data collected were treated and analyzed using the software SPSS Statistics v.22 (IBM, USA). To analyze the effects of caffeine on sperm motility, a general linear model with the Bonferroni correction was used $(p<0.05)$. One-way ANOVA $(p<0.05)$ was performed followed by Student-Newman-Keuls (S-N-K) as a post hoc test for the motility, viability and fertilization tests after Portuguese oyster sperm cryopreservation and in toxicity studies. Results are expressed as a mean of percentages in all analyses of 3-5 individual males.

\section{Results}

\subsection{Effect of the extender composition}

Two methods were tested in this assay in order to check the long term effect of sperm dilution prior to cryopreservation. Preliminary tests were done to check the 
necessity of sperm acclimation to RT prior to being used when previously stored at $4^{\circ} \mathrm{C}$. It was shown that $C$. angulata sperm can be stored successfully at $4 \stackrel{\text { ㄷ }}{ }$ for 3 days without losing motility and that acclimation at room temperature prior to the motility analysis enhances $(p<0.05)$ the percentage of motile cells (Figure 1 supplementary data). Incubation with caffeine did not stimulate an increase in the percentage of motile cells (Figure 1 supplementary data, $p<0.05$ ).

\subsection{Toxicity test}

In general, exposure to CPAs decreased sperm motility. The effect of caffeine supplementation depended on the CPA used. It significantly increased motility percentages in: 10 and 20\% PEG, 5\% DMSO and 5\% MetOH $(p<0.05)$. The other conditions tested showed no significant differences between the samples exposed to CPAs and the respective fresh control (Figure 1). The CPA with the least toxic effect in terms of motility was the 10\% PEG diluted in Asw supplemented with caffeine (Figure 1A) and $20 \%$ MetOH was the most toxic when diluted in both mediums (Figure 1C) ( $p<$ $0.05)$.

With regard to sperm viability, caffeine supplementation in the dilution medium did not have any drastic effects on cell viability. Also, as expected, 5\% CPA concentration showed higher viability values for all the CPAs used in both dilution mediums (Figure 2), and the most harmful conditions were $20 \% \mathrm{MetOH}$ in terms of cell survival rate (Figure $2 \mathrm{D})(\mathrm{p}<0.05)$. In view of these results, MetOH was discarded in the next step of the cryopreservation trials.

Linking the results for motility and viability, the toxicity test defined $10 \%$ PEG as the least toxic combination for $C$. angulata spermatozoa (viable sperm $>85 \%$ ), as no 
significant differences were observed in either parameter (motility and viability) when compared with fresh sperm.

\subsection{Cryopreservation assays}

\subsubsection{DMSO using a faster freezing method reported the highest viability rates.}

Two CPAs were chosen according to the previous results and to literature references for Crassostrea genus. Therefore, PEG was selected as the least toxic CPA in terms of sperm viability and motility and DMSO was selected in terms of producing a reduction in sperm agglutination after post-thawing, a process described by several authors in oyster sperm cryopreservation and confirmed in this study (lerepoli et al., 2004, Hassan et al., 2015).

At the slower freezing rate, post-thaw sperm motility was very low $(<5 \%)$ in all treatments and significantly different from the control $(p<0.05)$ (Figure 3A). No significant differences were observed between CPAs, their concentrations or extender mediums. The highest viability rates (>40\%) were obtained using 10 and $20 \%$ DMSO and $10 \%$ PEG, regardless of the extender used (with and without caffeine) (Figure 3B).

The same motility results were observed at the faster freezing rate. Sperm motility after cryopreservation was very low in comparison with the fresh control $(>40 \%)$ (Figure 4A). These motility results were independent from the conditions tested (CPAs, their concentrations and dilution medium) and no significant differences were observed between the cryopreserved samples. According to the viability results, higher viability rates were obtained using 10 and $20 \%$ DMSO (with and without caffeine as additive) (Figure 4B). The viability rates were, in all cited cases, higher than $70 \%$ and no significant differences were observed in comparison with the fresh control (Figure 4B). 
The results of the agglutination test coincided with previously described results. The use of PEG increased the agglutination level in sperm when compared with DMSO (Figure 2, supplementary material) and the respective images taken of the viability test confirmed that most of the cells cryopreserved with PEG were all non-viable (stained with PI, red cells) (Figure 2, supplementary material), contrary to the results obtained using DMSO (SYBR green positive cells).

In summary, PEG proved to be inadequate for oyster sperm cryopreservation, since it produced the worst results in terms of agglutination and sperm viability in both freezing rates and at the two concentrations used (Figures $3 \mathrm{~B}$ and $4 \mathrm{~B}$ ). However, regardless of the results obtained in the toxicity assays, $10 \%$ DMSO diluted in Asw (Figure 4B) was the best CPA to use with a faster freezing rate.

\subsubsection{The type of container was not critical for sperm viability and motility after}

\section{cryopreservation.}

There were no significant differences in terms of sperm post-thaw motility and viability between samples stored in $0.5 \mathrm{~mL}$ French straws and $1 \mathrm{~mL}$ cryovials. Motility values were lower in cryopreserved sperm compared with fresh (Figure 5A). However, no significant differences were found in terms of cell viability (Figure 5B). Taking into account that no significant differences were found among these containers, we chose the straws as they were the best and most economical way to store and transport large quantities of these cells.

\subsubsection{Fertilization ability is the definitive assay to ensure sperm quality after} cryopreservation. 
Taking into account the low motility rates, a fertilization assay was necessary to demonstrate $C$. angulata sperm functionality. The fertilization trials performed with four different spermatozoa/egg ratios revealed significant differences between the different conditions. The fresh sperm, as expected, showed the highest percentage (>90\%) of fertilized eggs (Figure 6). As shown in Figure 6, higher fertilization rates (58$72 \%$ ) were obtained, ranging from 2.500 to $10000 \mathrm{spz} / \mathrm{egg}$, the highest ratio being the one that showed better results (>70\%), even if not significantly different from the rest.

\section{Discussion}

C.angulata sperm showed motility for 3 days without decreasing significantly. These observations are in accordance with previous studies carried out in oyster sperm motility (Rose and Heath, 1978) which demonstrated that sperm movement usually lasts longer in marine invertebrates than in marine fish species. Sperm motility lasted 4 to 5 hours in Eastern oyster (C. virginica) (Faure, 1996) and $>24 \mathrm{~h}$ in Pacific oyster (C. gigas) (Boulais et al., 2015). C. angulata sperm proved to be very resistant and to have long duration movement, since motility did not decrease until the third day (Figure 1, supplementary material). Long-term sperm motility duration can be attributed to the reproductive strategy of the different species. According to published literature, many parameters can influence sperm motility, such as temperature and extender media composition including additives such as caffeine (Suquet et al., 2012). For this reason, we evaluated the influence of several parameters to obtain the best possible sperm conditions before sperm cryopreservation and to determine standard conditions for sperm motility analysis. In our results, sperm motility significantly increased after 
refrigerated sperm acclimation to room temperature for 30 minutes (Figure 1, supplementary material). This motility temperature influence could be explained by an increase in the metabolism of these cells at higher temperatures, triggering the activation of non-motile cells. Like in humans and other species, when spermatozoa are incubated at lower temperatures than the natural fertilization conditions, they adopt a resting state, which allows sperm to preserve their energy (Gallup, 2009). This mechanism is known as "the activation hypothesis". This hypothesis possibly explains the diminished motility of refrigerated spermatozoa compared to those acclimated to RT.

The addition of caffeine to the dilution medium has been widely studied in fish species such as Pacu (Piaractus mesopotamicus), where sperm motility in fresh samples increased with a linear correlation with the increase in caffeine concentration (Carvalho et al., 2014). In Pacific oyster, Suquet and colleagues (2012) reported that the sperm maturation process could be stimulated through the addition of $10 \mathrm{mM}$ caffeine to sea water, increasing the beat frequency of flagella. This reaction can also be obtained with the addition of theophylline (Robles et al., 2003). Caffeine and theophylline are methylxanthines that act as phosphodiesterase inhibitors and can increase the intracellular cAMP (a derived form of ATP), which stimulates protein phosphorylation and triggers sperm movement (Suquet et al., 2012). As a consequence of the action of methylxanthine, we can assume that its addition to the activation solution should be similar to the addition of AMPc. In our study, the effect of caffeine supplementation was beneficial during the toxicity assays. However, when we carried out different cryopreservation assays (with two different freezing rates) using PEG and 
DMSO with and without $10 \mathrm{mM}$ caffeine, we found that the addition of caffeine to PEG caused sample agglutination (Figure 2, supplementary material). Sperm agglutination is a phenomenon that appears in sperm solutions after thawing and has previously been described in other oyster species such as Pacific and Eastern oyster (Dong et al., 2007). Moreover, the freezing rate also played an important role in this phenomenon, since agglutination was higher in samples cryopreserved with a slower freezing rate and the use of faster freezing was avoided. These results are similar to those in previous studies by Dong et al., (2007), demonstrating that CPAs at low concentrations, cooling at slow rates and high sperm concentration led to the formation of higher levels of agglutination. Agglutination drastically affected C.angulata sperm viability and helped to explain the fact that toxicity studies do not take into account the possible interactions of CPAs with the following step of the cryopreservation process. For all these reasons, toxicity assays are a previous step that should be corroborated with a cryopreservation trial before discarding or choosing any condition. Thus, in the present study, although 10\% PEG was the CPA with the least toxic effect on the cells (Figure 1 and 2), 10-20\% DMSO proved to have a better CPA effect. DMSO is one of the most suitable CPAs due to its ability to penetrate cells rapidly, thus protecting cells from freezing and thawing injury (Hassan et al., 2015).

Taking into account that motility rates are lower in cryopreserved samples compared to fresh samples, a fertility assay was included to demonstrate sperm functionality after the selected cryopreservation protocol (10\% DMSO, the faster freezing rate and $0.5 \mathrm{~mL}$ straws as a container). Correlations between motility and fertilization capacity have been found in different oyster species (Suquet et al., 2010). 
However, some studies report that low motility cryopreserved sperm is able to fertilize, as is the case of the Eastern oyster, C. virginica, described by Yang et al., (2012). Similar to this previous study, in our results, post-thaw motility did not show correlation with fertility. Oyster sperm can swim continuously for hours upon suspension in seawater (considerably longer than for sperm of most fishes), providing an advantage in fertilization ability. As a consequence, post-thaw motility is not always effective at predicting fertilizing ability and this fact was previously reported by other authors in oyster sperm (Paniagua-Chávez et al., 2006). Although motility and structural integrity are important sperm quality assessment indicators, theoretically, fertilization trials should be the most unequivocal method for determining sperm quality. According to studies performed in C. gigas by Adams et al., (2004), the fertilization ability of fresh sperm was 30 - to 100 -fold higher than that of sperm cryopreserved with DMSO. According to Hassan et al., (2015) in several other oyster species, 1000 to 10000 post-thaw spermatozoa per egg is enough to produce similar fertilization rates as those obtained using fresh sperm, if sperm functionality was not affected by cryopreservation. Based on these results, we selected the sperm per egg ratios in the present study obtaining fertilization percentages higher than $70 \%$ when using 10000 spermatozoa/egg. The results obtained in this work can be comparable to others using a similar protocol developed for C. virginica (10\% DMSO, 20 minutes equilibration time, fast freezing rate, use of straws, thawing at $40{ }^{\circ} \mathrm{C}$ for $8 \mathrm{~s}$ ), where the authors achieved fertilization rates of 77\% (Yang et al., 2012).

To summarize, in C. angulata sperm cryopreservation, CPA toxicity tests are important to discard some CPAs and concentrations, but are not definitive. We observed that agglutination is also a significant problem for C. Angulata sperm 
cryopreservation, this phenomenon being avoided by using some CPAs and discarding some conditions (slow freezing). In our study, fertilization assays were the definitive tool to demonstrate the functionality of the cryopreservation protocol. In the future, other quality assays should be performed in this species to check for cryopreservation effects at different levels with the aim of guaranteeing the total safety of the technique.

\section{Conclusions}

- Toxicity tests do not take into account the following steps of the cryopreservation protocol as agglutination phenomenon.

- $10 \%$ DMSO, the faster freezing rate and the use of straws as containers are the best cryopreservation conditions for Portuguese oyster sperm.

- Fertilization ability is the definitive tool for evaluating the efficiency of a cryopreservation protocol efficiency in oyster sperm.

- A sperm cryopreservation protocol was successfully established in C.angulata for the first time.

\section{Acknowledgments}

The authors are grateful to Marcelo Livramento (CCMAR) and Ana Margarete Ramos (IPMA) for their technical assistance during oyster sampling and oyster conditioning. This work was supported by project CRIOBIV-31-03-05-FEP-59. Marta 
F Riesco was supported by a postdoc contract supported by CRIOBIV project. The authors would like to thank COST action (Food and Agriculture) AQUAGAMETE (FA1205) and the oyster farms that supplied the biological material: Antonio Falcão, (Sociedade Agrícola Herdade das Moitas) and Acácio José Guerreiro Simões.

\section{References}

-Adams, S. L., Smith, J. F., Roberts, R. D., Janke, A. R., Kaspar, H. F., Tervit, H. R., Pugh, P. A., Webb, S. C., King, N. G., 2004. Cryopreservation of sperm of the Pacific oyster (Crassostrea gigas): development of a practical method for commercial spat production. Aquaculture 242, 271-282.

-Boudry, P., Heurtebise, S., Collet, B., Cornette, F., Gérard, A., 1998. Differentiation between populations of the Portuguese oyster, Crassostrea angulata (Lamark) and the Pacific oyster, Crassostrea gigas (Thunberg), revealed by mtDNA RFLP analysis. Journal of Experimental Marine Biology and Ecology 226, 279-291.

-Boulais, M., Soudant, P., Le Goic, N., Quéré, C., Boudry, P., Suquet, M., 2015. Involvement of mitochondrial activity and OXPHOS in ATP synthesis during the motility phase of spermatozoa in the Pacific Oyster, Crassostrea gigas. BOR. 93, 118,1-7.

-Carvalho, A. F. S., Machado, M. R. F., Andrade, E. S.; Murgas, L. D. S., Zangeronimo, M. G., 2014. Effect of Caffeine Added to the Activating Solution on Sperm Motility of Fresh and Thawed Semen of Pacu, Piaractus mesopotamicus, and Curimba, Prochilodus lineatus. Journal of the World Aquaculture Society 45, 75-81. 
-Comps, M., Bonami, J. R., Vago, C., Campillo, A., 1976. Une virose de I'huitre Portugaise (Crassostrea angulata Lmk). Comptes Rendus de I'Academie des Sciences, Série 3, Sciences de la Vie 282, 1991-1993.

-Corporeau, C., Vanderplancke, G., Boulais, M., Suquet, M., Quéré, M., Boudry, P., Huvet, A., Madec, S., 2012. Proteomic identification of quality factors for oocytes in the Pacific oyster Crassostrea gigas. Journal of Proteomics 75, 5554-5563.

-Dong, Q., Eudeline, B., Huang, C., Allen S. K. J., Tiersch, T. R., 2005. Commercial-scale sperm cryopreservation of diploid and tetraploid Pacific oysters, Crassostrea gigas. Cryobiology 50, 1-16.

-Dong, Q., Huang, C., Tiersch, T. R., 2007. Control of sperm concentration is necessary for standardization of sperm cryopreservation in aquatic species: Evidence from sperm agglutination in oysters. Cryobiology 54, 87-98.

-Dong, Q., Tiersch, C., Huang, T.R., 2011. The need for standardization in cryopreservation: a case study with oysters. In: Tiersch, T.R., Green, C.C. (Eds.), Cryopreservation in Aquatic Species, 2nd edition World Aquaculture Society, Baton Rouge, Louisiana, pp. 581-593.

-EFSA, European Food Safety Authority., 2015. Oyster mortality. EFSA Panel on Animal Health and Welfare (AHAW). EFSA Journal 13, 4122.

-Faure, C., 1996. Paramètres physiologiques de l'émission et de l'activation des gamètes mâles de deux mollusques bivalves: la coquille Saint Jacques Pecten maximus (L.) et l'huîtrecreuse Crassostrea gigas (Thunberg). Thesis. University of Paris 7; 255 p. 
- Gallup, G.G., 2009. On the origin of descended scrotal testicles: the activation hypothesis. Evol. Psychol 7, 517-526.

-Gwo, J. C., Chen, C. W., Cheng, H. Y., 2002. Semen cryopreservation of small abalone (Haliotis diversicolor supertexa). Theriogenology 58, 1563-1578.

-Hassan, M. M., Qina, J. G., Li, X., 2015. Sperm cryopreservation in oysters: A review of its current status and potentials for future application in aquaculture. Aquaculture 438, 24-32.

-Hu, E., Yang, H., Tiersch, T.R., 2011. High-throughput cryopreservation of spermatozoa of blue catfish (Ictalurus furcatus): establishment of an approach for commercial-scale processing. Cryobiology 62, 74-82

-leropoli, S., Masullo, P., Espirito Santo, M., Sansone, G., 2004. Effects of extender composition, cooling rate and freezing on the fertilisation viability of spermatozoa of the Pacific oyster (Crassostrea gigas). Cryobiology 49, 250-257.

-Paniagua-Chávez, C.G., Jenkins, J., Segovia, M., Tiersch, T.R., 2006. Assessment of gamete quality for the eastern oyster (Crassostrea virginica) by use of fluorescent dyes. Cryobiology 53, 128-38.

-Robles, V., Cabrita E., Cuñado, S., Herráez, M.P., 2003. Sperm cryopreservation of sexreversed rainbow trout (Oncorhynchus mykiss): parameters that affect its ability for freezing. Aquaculture 224, 203-212.

-Rose, C. D., Heath, E., 1978. Viability of American oyster, Crassostrea virginica, spermatozoa exposed to stress. Estuaries 1, 245-251. 
-Song, Y. P., Suquet, M., Quéau, I., Lebrun, L., 2009. Setting of a procedure for experimental fertilization of Pacific oyster (Crassostrea gigas) oocytes. Aquaculture 287, 311-314.

-Suquet, M., Labbé, C., Brizard, R., Donval, A., Le Coz, J. R., Quere, C., Haffray, P., 2010. Changes in motility, ATP content, morphology and fertilization capacity during the movement phase of tetraploid Pacific oyster (Crassostrea gigas) sperm. Theriogenology 74, 111-117.

-Suquet, M., Cosson, J., Donval, A., Labbé, C., Boulais, M., Haffray, P., Bernard, I., Fauvel, C., 2012. Marathon vs sprint racers: an adaptation of sperm characteristics to the reproductive strategy of Pacific oyster, turbot and seabass. J. Appl. Ichthyol. 28, 956-960.

-Suquet, M., Labbé, C., Puyo, S., Mingant, C., Quittet, B., Boulais, M., Queau, I., Ratiskol, D., Diss, B., Haffray, P., 2014. Survival, Growth and Reproduction of Cryopreserved Larvae from a Marine Invertebrate, the Pacific Oyster (Crassostrea gigas). PLoS ONE 9, e93486.

-Tiersch, T.R., 2000. Introduction. In: Tiersch, T.R., Mazik, P.M. (Eds.), Cryopreservation in

Aquatic Species. World Aquacult. Soc., Baton Rouge, Louisiana, pp. xix-xxvi.

-Yang, H., Supan, J., Guo, X., Tiersch, T. R., 2013. Nonlethal Sperm Collection and Cryopreservation in the Eastern Oyster (Crassostrea virginica). Journal of Shellfish Research 32, 429-437. 


\section{Figure legends}

Figure 1. C. Angulat motility results after toxicity tests. Four different cryoprotectants (CPAs) were tested: A)polyethylene glycol (PEG), B)dimethyl sulfoxide (DMSO), C)methanol (MetOH) and D) ethylene glycol (EG), at three final concentrations $(5 \%, 10 \%$ and $20 \%)$ in two dilution mediums: Artificial sea water (Asw) and Asw supplemented with $10 \mathrm{mM}$ caffeine. Pre-diluted fresh sperm (Asw or Asw+ $10 \mathrm{mM}$ caffeine) was included as the control. All results were expressed as a mean of percentages \pm SE of 3 individual males. One-way ANOVA $(p<0.05)$ was performed followed by Student-Newman-Keuls (SNK) as a post hoc test. Different letters show significant differences between conditions $(p<0.05)$.

Figure 2. C. Angulata viability results after toxicity tests. Four different cryoprotectants (CPAs) were tested: A) polyethylene glycol (PEG), B) dimethyl sulfoxide (DMSO), C) methanol (MetOH) and D) ethylene glycol (EG), at three final concentrations (5\%, $10 \%$ and $20 \%$ ) in two dilution mediums: Artificial sea water (Asw) and Asw supplemented with $10 \mathrm{mM}$ caffeine. All results were expressed as a mean of percentages \pm SE of 4 individual males. Pre-diluted fresh control sperm was included (Asw or Asw $10 \mathrm{mM}$ caffeine). One-way ANOVA $(p<0.05)$ was performed followed by Student-Newman-Keuls (SNK) as a post hoc test. Different letters show significant differences between conditions $(p<0.05)$. 
Figure 3. C. Angulata motility and viability results after slower freezing. Two cryoprotectants, polyethylene glycol (PEG) and dimethyl sulfoxide (DMSO), at 10 and $20 \%$ were tested and compared with the pre-diluted fresh control (1:10 in Asw or Asw + $10 \mathrm{mM}$ caffeine). Motility (A) and viability (B) were expressed as a mean of percentages \pm SE of 4 individual males. One-way ANOVA $(p<0.05)$ was performed followed by Student-Newman-Keuls (SNK) as a post hoc test. Different letters show significant differences between conditions $(p<0.05)$.

Figure 4. C. Angulata motility and viability results after faster freezing. Two cryoprotectants, polyethylene glycol (PEG) and dimethyl sulfoxide (DMSO), at 10 and $20 \%$, were tested and compared with the pre-diluted fresh control (1:10 in Asw or Asw $+10 \mathrm{mM}$ caffeine). Motility (A) and viability (B) were expressed as a mean of percentages \pm SE of 4 individual males. One-way ANOVA $(p<0.05)$ was performed followed by Student-Newman-Keuls (SNK) as a post hoc test. Different letters show significant differences between conditions $(p<0.05)$.

\section{Figure 5. C.angulata motility and viability results comparing two containers,} straws and cryovials. The best condition obtained after cryopreservation: $10 \%$ DMSO in Asw, using the faster freezing method, was tested and compared with the pre-diluted fresh control (Asw). Motility (A) and viability (B) were expressed as a mean of percentages \pm SE in 4 individual males. One-way ANOVA $(p<0.05)$ was 
performed followed by Student-Newman-Keuls (SNK) as a post hoc test. Different letters show significant differences between conditions $(p<0.05)$.

Figure 6. C angulata fertilization results comparing fresh and cryopreserved sperm using different fertilization rates. Percentage of fertilized eggs using fresh and cryopreserved sperm. Four different ratios spermatozoa/egg (number of males per pool=3, number of pools=5); mean $\pm \mathrm{SE}$ ) were used for the thawed sperm. Oneway ANOVA $(p<0.05)$ was performed followed by Student-Newman-Keuls $(S N K)$ as a post hoc test. Different letters show significant differences between conditions ( $p$ $<0.05)$.

\section{Supplementary material}

Figure 1, supplementary material. Preliminary tests in C. Angulata to test the effect of caffeine and room temperature incubation on sperm motility. A) Total sperm motility during 3 days, for pre-diluted sperm (1:10) in artificial sea water (Asw) or Asw supplemented with $10 \mathrm{mM}$ caffeine. B) Total sperm motility during 3 days, for pre-diluted sperm (1:10) in Asw measured at 4ํㄷ or at room temperature (RT) after 30 min incubation. All results were expressed as a mean of percentages \pm SE in 4 individual males. Different letters show differences between treatments (General linear model, Bonferroni, $p<0.05$ ).

Figure 2, supplementary material. C. angulata agglutination results. DMSO and PEG were compared at the same concentration to test agglutination comparing to 
viability results. Photos of thawed sperm, and of viability, after staining with propidium iodide (PI) and SYBR-green. Cryopreservation was performed at a fast freezing rate using $10 \%$ PEG and $10 \%$ DMSO in two dilution mediums, Asw and Asw+caffeine. 
ACCEPTED MANUSCRIPT

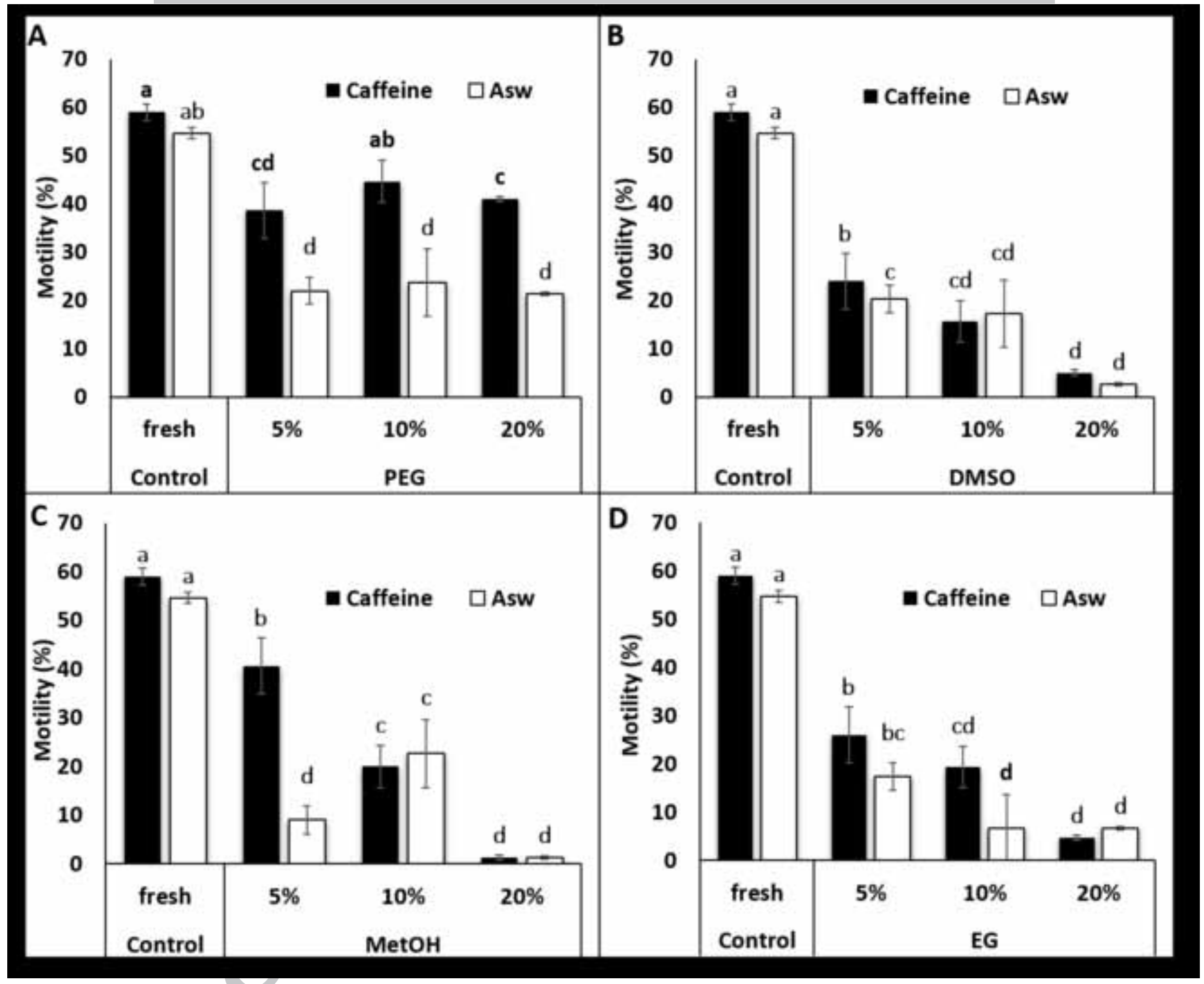


ACCEPTED MANUSCRIPT

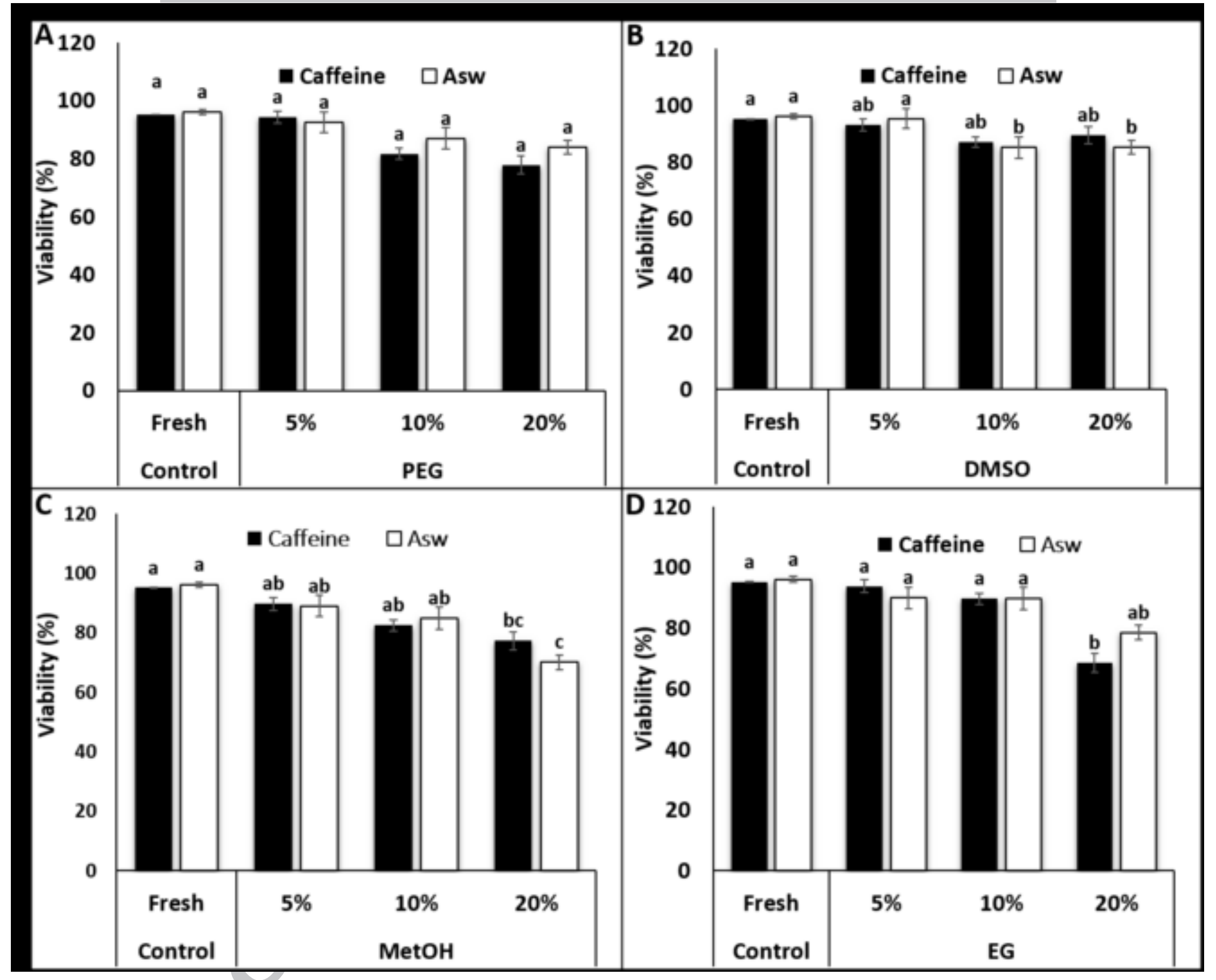




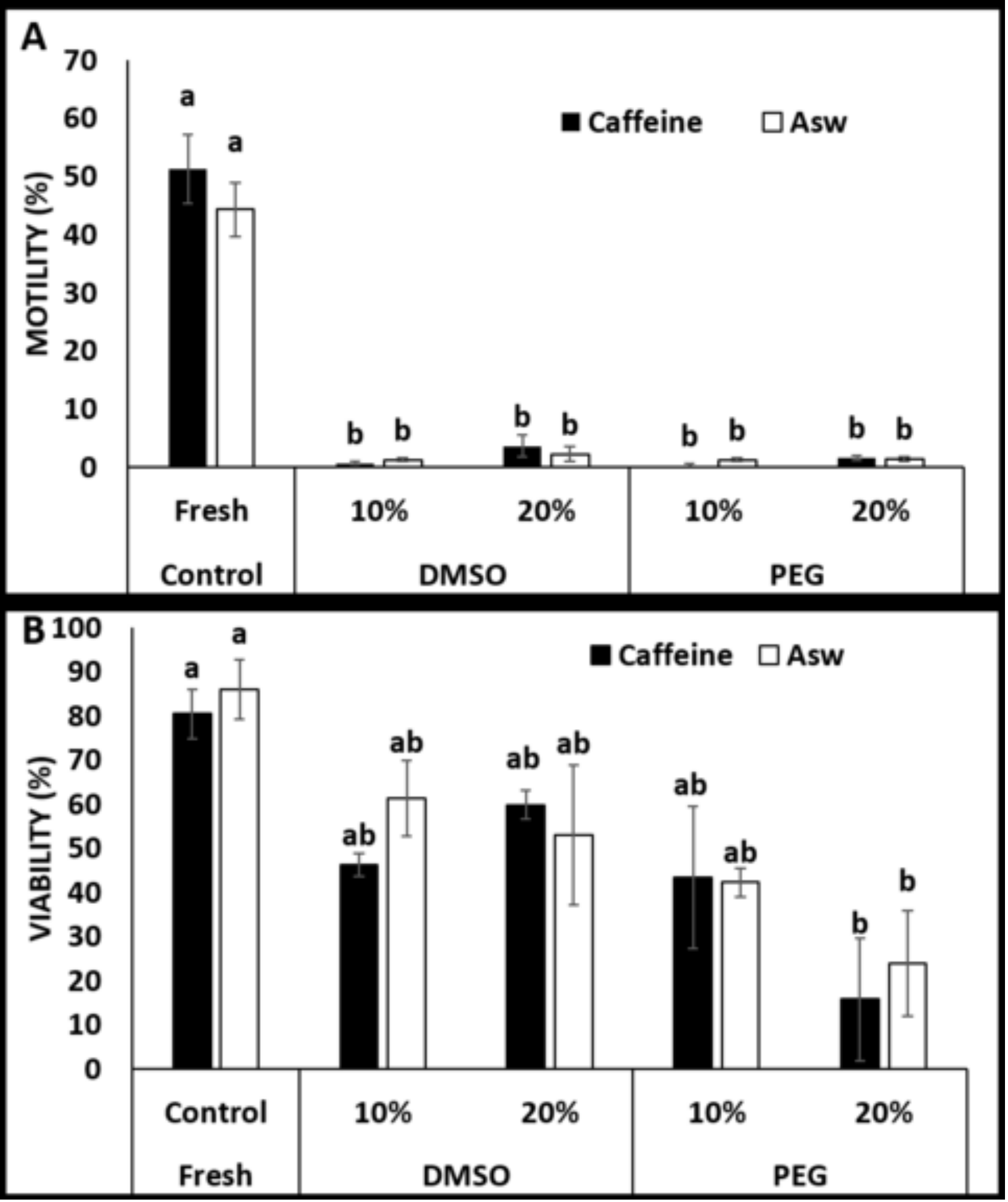



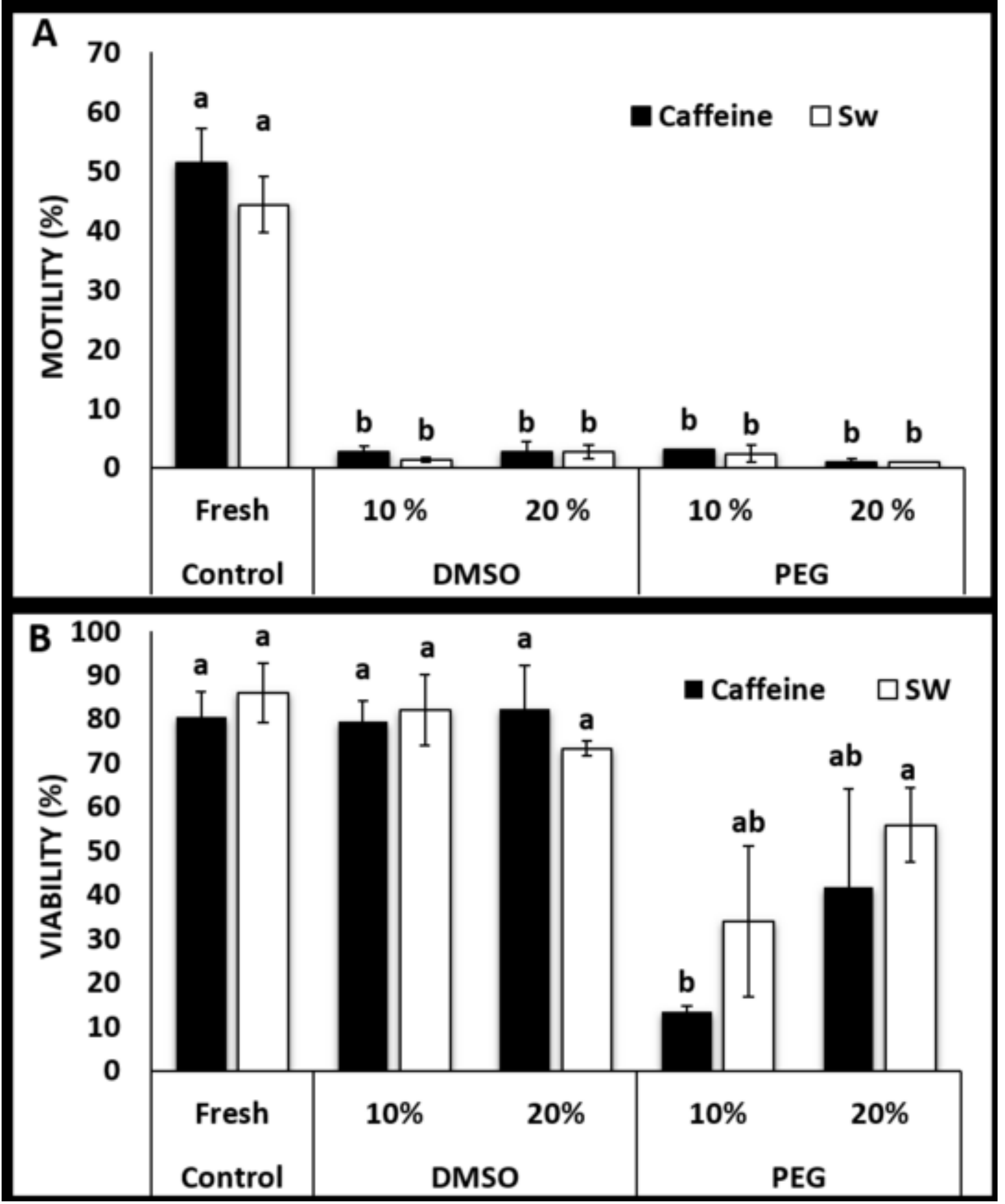


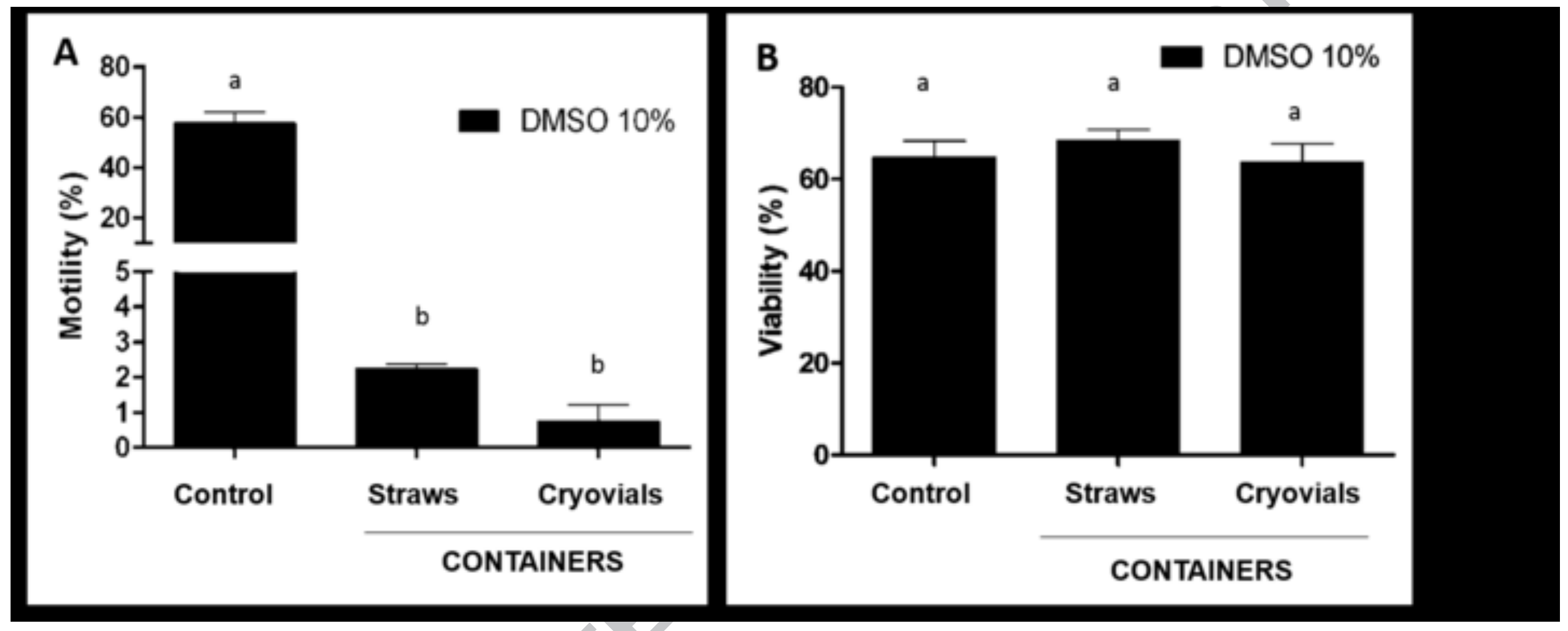




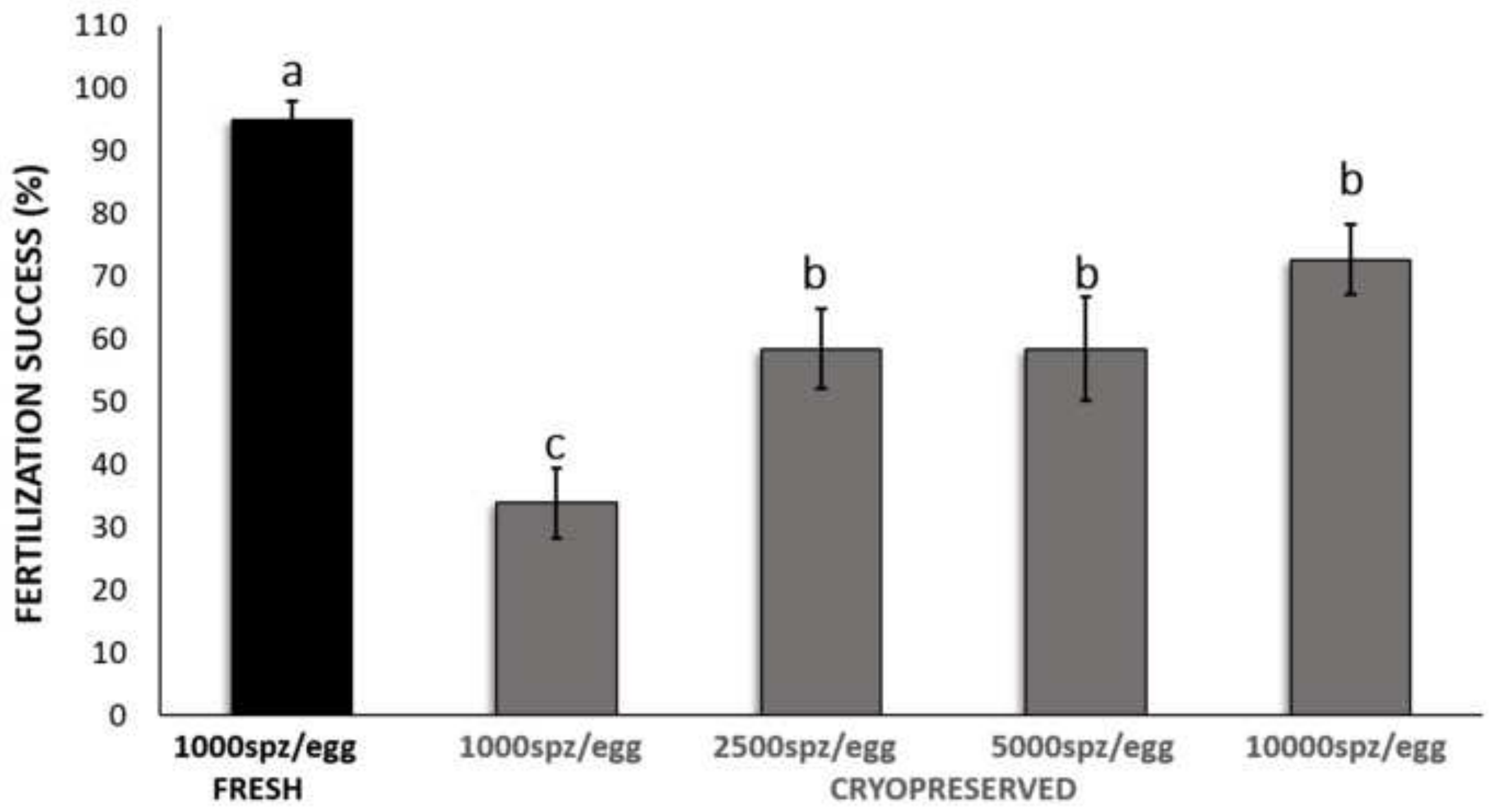

